\title{
Dual source populations of substorm-associated ring current ions
}

\author{
M. Yamauchi ${ }^{1}$, Y. Ebihara ${ }^{2}$, I. Dandouras ${ }^{3}$, and H. Rème ${ }^{3}$ \\ ${ }^{1}$ Swedish Institute of Space Physics, Kiruna, Sweden \\ ${ }^{2}$ Institute for Advanced Research, Nagoya University, Nagoya, Japan \\ ${ }^{3}$ Centre d'Etude Spatiale des Rayonnements, Toulouse, France
}

Received: 7 October 2008 - Revised: 15 January 2009 - Accepted: 28 February 2009 - Published: 1 April 2009

\begin{abstract}
Sources of low-energy ring current ions in the early morning sector (eastward drifting energy domain of about $<5 \mathrm{keV}$ ) are examined using both statistical analyses and numerical tracing methods (phase-space mapping and simulation). In about $90 \%$ of Cluster perigee traversals at 02 07 local time, these low-energy ring current ions have dual ion populations: one is wedge-like energy-dispersed ions, and the other is a band-like ions over different latitudes in a narrow energy range at the upper energy threshold of the wedge-like energy-dispersed ions. Both components are most likely created during past substorm activities. Numerical tracing results strongly suggest that these two components have different sources with different temperatures and elapsed times. The band-like part most likely comes from ions with plasma sheet temperature $(\sim 1 \mathrm{keV})$, and the energy-dispersed part most likely comes from cold ions (temperature $<0.1 \mathrm{keV}$ ). The source density of the cold component $\left(0.2 \sim 0.5 \times 10^{6} / \mathrm{m}^{3}\right)$ is slightly less than that of the hot component $\left(0.5 \times 10^{6} / \mathrm{m}^{3}\right)$, while Cluster observation shows slightly higher density for the wedge-like part than the low-energy band-like part. The hot source component also explains the observed high-energy $(>10 \mathrm{keV})$ ions drifting westward after adiabatic energization in the nightside under time-varying electric field. The wedge-like part has much shorter elapsed time, i.e., less charge-exchange loss, than the band-like part.
\end{abstract}

Keywords. Magnetospheric physics (Energetic particles, trapped; Storms and substorms) - Space plasma physics (Charged particle motion and acceleration)

Correspondence to: M. Yamauchi (m.yamauchi@irf.se)

\section{Introduction}

Ion motions in the inner magnetosphere (region of nearly dipole magnetic field) are primarily governed by energydependent magnetic (gradient-B and curvature) drift and energy-independent $\boldsymbol{E} \times \boldsymbol{B}$ drift. The magnetic drift influences mainly the high-energy ions and moves them mainly westward at all local time. The $\boldsymbol{E} \times \boldsymbol{B}$ drift influences mainly low-energy ions and moves them mainly sunward, i.e., westward in the evening sector and eastward in the morning sector (Alfvén and Fälthammar, 1963; Lennartsson et al., 1979). Therefore, the threshold energy that demarcates the westward drift and the eastward drift decreases with local time from the morning sector to the evening sector (Quinn and McIlwain, 1979). In the morning sector at 65 degree Inv., the statistical threshold energy ("ion gap" in their terminology) is $5 \sim 8 \mathrm{keV}$ (Kovrazhkin et al., 1999). During magnetic storms, this energy increases due to the enhanced electric field.

In the eastward drifting energy domain, wedge-like energy-dispersed sub-keV ion structure, so called the wedgelike structure, is widely observed in the morning to noon sectors (Quinn and McIlwain, 1979; Sauvaud et al., 1981; Newell et al., 1986; Yamauchi et al., 1996, 2005; Ebihara et al., 2001). In Viking and Cluster observation, the wedgelike structure is classified into three major energy-latitude dispersion patterns, increasing energy with latitude ("ordinary"), decreasing energy with latitude ("reversed"), and decreasing energy toward both high latitude and low latitude from a certain latitude ("bridge-like"). Figure 1a shows an example of "ordinary" and "bridge-like" wedge-like structures observed in the noon sector. Both statistics and case studies for $>0.1 \mathrm{keV}$ part show that the wedge-like structure is formed in the night-to-morning sector during high $\mathrm{AE}$ activity, moves eastward with about $0.5 \sim 2 \mathrm{MLT} /$ hour drift velocity, and decays with a life time about several to ten hours (Yamauchi and Lundin, 2006; Yamauchi et al., 2006).

Published by Copernicus Publications on behalf of the European Geosciences Union. 


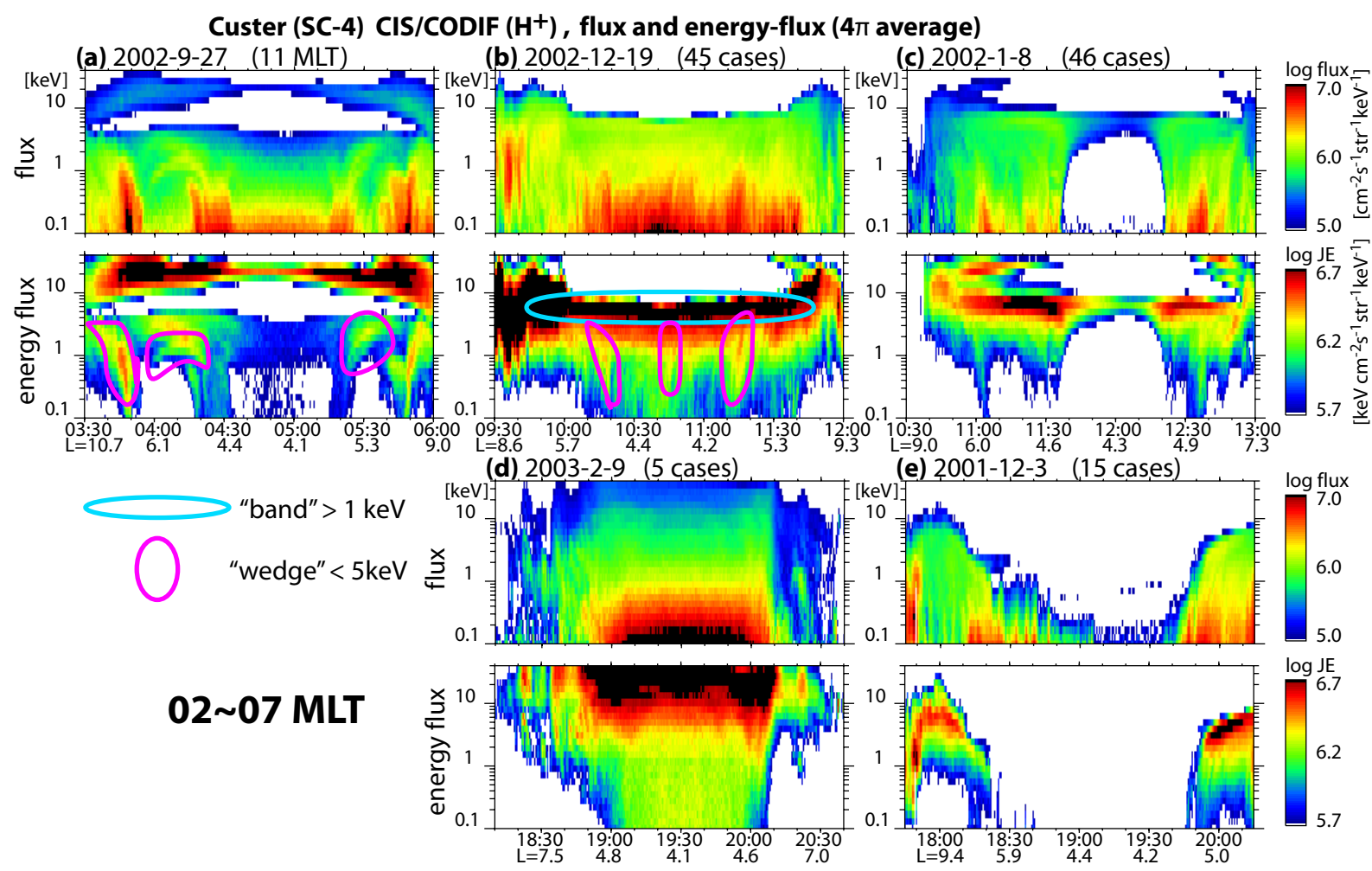

Fig. 1. Energy-time spectrograms of $4 \pi$ averaged proton flux (upper) and energy flux (lower) observed by Cluster spacecraft-4 CIS/CODIF at $0.1-40 \mathrm{keV}$. Five traversals (150 min each) are displayed from (a) $11 \mathrm{LT}$ and (b) (e) $02 \sim 07 \mathrm{LT}$. The spacecraft location is given by L value. The uniform background count that peaks near the middle part (04:30 05:20 UT in (a), 10:20 11:20 UT in (b), and 19:00 20:00 UT in (d)) is contamination from $\mathrm{MeV}$ particles of the radiation belt (they penetrate through the walls of the instrument). Since this contamination is independent of measurement energy, it appears uniform in the energy flux and counter proportional to the energy in the flux. The band-like structure is marked by blue line and the wedge-like energy-latitude sub-keV ion structure is marked by purple line.

Ebihara et al. (2001) performed the first simulation to reconstruct three major dispersion patterns and their local time dependences observed by Viking. This simulation assumed an ion source of wide energy range (5 keV temperature) at $L=10$, and did not quantitatively derive the source energy distribution (density and temperature). Although the Viking observation of the wedge-like structure is mainly in the sub$\mathrm{keV}$ energy domain, one cannot assume sub-keV source temperature because the observed signature has experienced the drift which works as an energy filter (passing only low energy for the eastward drift and only high energy for the westward drift). The upper energy limit of the wedge-like structure depends on the electric field strength but not to the source temperature. This is why the $5 \mathrm{keV}$ source in the plasma sheet could reproduce the wedge-like structure in the simulation of Ebihara et al. (2001). In fact, the observed energy extent of the wedge-like structure increases to more than $1 \mathrm{keV}$ (sometimes to $\sim 10 \mathrm{keV}$ ) in the morning sector which is close to the source region (Yamauchi et al., 2006).

To reconstruct the source temperature, one needs a good estimate of electric field during the drift as well as a satellite ion data with good sensitivity at wide energy range. For this purpose, Ebihara et al. (2008) applied the same particle simulation on the wedge-like structure observed by the Equator-S satellite. The reconstructed result is somewhat unexpected: the wedge-like structure most likely originates from cold ions (about $100 \mathrm{eV}$ temperature) instead of hot plasma sheet ions. Since the drift history depends on the electric field model, this result needs confirmation.

The different source temperature of the wedge-like structure from the hot plasma sheet is consistent with the particle drift simulation of the multiple band-like structures by Vallat et al. (2007). They explained the double bands of ions in the Cluster data (21 November 2001 event) as combination of a westward drifting ion band and an eastward drifting ion band. However, they could not reproduce the wedge-like structure that extended from the second band toward low energy, indicating that the wedge-like structure has a different source from the eastward drifting band-like structure.

In the Cluster perigee observation in the morning sector, many traversals show simultaneous existence of the bandlike structure at around several $\mathrm{keV}$ and the wedge-like structure extending from the band-like structure toward low energy, both overlapping to each other (Yamauchi et al., 2006). 
However, these observations are not sufficient to confirm the dual sources by themselves because all stripes of the wedge-like structure have the same upper energy limit according to the drift theory. Since Cluster orbit does not cover inside $4.2 R_{E}$, the observed band-like structure could be a part of other stripes of the wedge-like structure that lie inside $4.2 R_{E}$. This was the interpretation in Yamauchi et al. (2006), in which these mixed case were classified as "extended $>1 \mathrm{keV}$ ".

If the eastward drifting wedge-like structure and the eastward drifting band-like structure have different sources, we have obvious questions: (1) relation to substorm activities of both components, (2) relative amount of both components in the source region. For the first question, one needs the same type of superposed epoch analyses as Yamauchi and Lundin (2006). For the second question, one needs to reproduce both components in a single simulation by assuming two ion populations in the source region: hot plasma sheet component for the band-like structure and cold component for the wedgelike structure. These two works are presented in this paper. In Sect. 2, we show Cluster statistics of correlation between the substorm activity and the morphology of the low-energy eastward drifting ring current ions. We use two year (2001 to 2003 spring) data from Cluster perigee traversals at 02 07 local time (LT). In Sect. 3, we show the numerical reconstruction of both the band-like structure and the wedge-like structure in a single simulation.

\section{Statistics}

The Cluster CIS experiment consists of two instruments for positive ion measurements from $\mathrm{eV}$ to tens $\mathrm{keV}$ : HIA without mass analyzer, and CODIF with time-of-flight mass analyzer. Unfortunately, HIA is often contaminated by radiation belt particles $(\mathrm{MeV}$ particles penetrate the instrument walls and directly hit the sensor) near perigee. Therefore, we use the CODIF data which is less contaminated by the radiation belt particles than the HIA data. Since the wedge-like structure in the $\mathrm{O}^{+}$channel or the $\mathrm{He}^{+}$channel are normally much less intense than that in the $\mathrm{H}^{+}$channel, we consider only $\mathrm{H}^{+}$ data in the present study. Details of the CIS/CODIF instrument are found in Rème et al. (2001).

We first classify the morphology of the energy-time spectrograms of energy flux. We chose 02 07 LT which show highest observation rate of the mixture of the band-like structure overlapping with the wedge-like structure. Since Cluster orbit is synchronous to the solar system, this local time corresponds to traversals from late October to late February. We examined traversals at 02 07 LT during three seasons (2001 to spring 2003, total 111 traversals). Figure $1 \mathrm{~b} \sim$ e shows typical spectrograms (flux and energy flux) from all four patterns. The number of traversals in each pattern is listed at the top of each spectrograms. For reference, we also show an
Table 1. Number of traversals classified by the peak AE during $6 \mathrm{~h}$ before the traversal.

\begin{tabular}{cccc}
\hline pattern & $<300 \mathrm{nT}$ & $300 \sim 500 \mathrm{nT}$ & $>500 \mathrm{nT}$ \\
\hline Figure 1d & 0 & 0 & 5 \\
Figure 1b & 4 & 8 & 33 \\
Figure 1c & 26 & 17 & 3 \\
Figure 1e & 10 & 1 & 4 \\
\hline
\end{tabular}

example of typical wedge-like structure observed in the noon sector in Fig. 1a.

Both Fig. 1b (one of 45 similar traversals) and Fig. 1c (one of 46 similar traversals) show mixture of the band-like structure and the wedge-like structure, with the difference in locations of the low-latitude cavity. If the structure extends to $L<4.2$, we observe it as continuous pattern like Fig. 1 b. If the innermost drifting shell of the structure move outside $L=4.5$, we observe it with cavity in the middle like Fig. 1c. We sometimes observe these patterns without cavity at spacecraft 1 (lower perigee altitude) and with cavity at spacecraft 4 (higher perigee altitude) simultaneously, and hence we primarily use spacecraft 4 for the present statistics. Figure 1c is one such example. Patterns represented by Fig. $1 \mathrm{~b}$ and $\mathrm{c}$ composes majority of the observation (91 out of 111 traversals), indicating that the band-like structure at several $\mathrm{keV}$ nearly always overlaps the wedge-like structure at this local time during solar maximum.

Figure 1d (one of 5 similar traversals) is similar to Fig. 1b but the energy of the band-like structure is located near the upper threshold of the CIS instrument. Figure 1e (one of 15 similar traversals) is similar to Fig. 1c but one cannot recognize the band-like structure at all. Patterns like Fig. 1c and e are observed during relatively low Kp geomagnetic conditions according to Vallat et al. (2007) who classified them as "nose", although this nose is not necessarily the same as traditional nose in the afternoon sector (e.g., Ejiri et al., 1980, and references therein).

We next examine the geomagnetic conditions for these 4 patterns. According Viking statistics (Yamauchi and Lundin, 2006), probability of observing the wedge-like structure at 05 07 MLT is about 80 90\% during first $4 \mathrm{~h}$ after the end of high AE activities. Therefore, we classify the geomagnetic activity by examining the peak $\mathrm{AE}$ index during past $6 \mathrm{~h}$ before the traversal. Since only provisional AE (not final $\mathrm{AE})$ from limited station is available, AE might be underestimated. Yet, we obtain a clear relation between the energylatitude morphology and $\mathrm{AE}$ as shown in Table 1.

The table indicates that recent high AE activity causes the band-like structure together with the wedge-like structure covering wide latitudinal range (Fig. $1 \mathrm{~b}$ and d). During quiet periods, the low-latitude limit of the structure moves outward (toward higher $L$ ), causing widening of the cavity (Fig. 1c 
CIS/CODIF H+ data (SC-4) , 2004-12-22

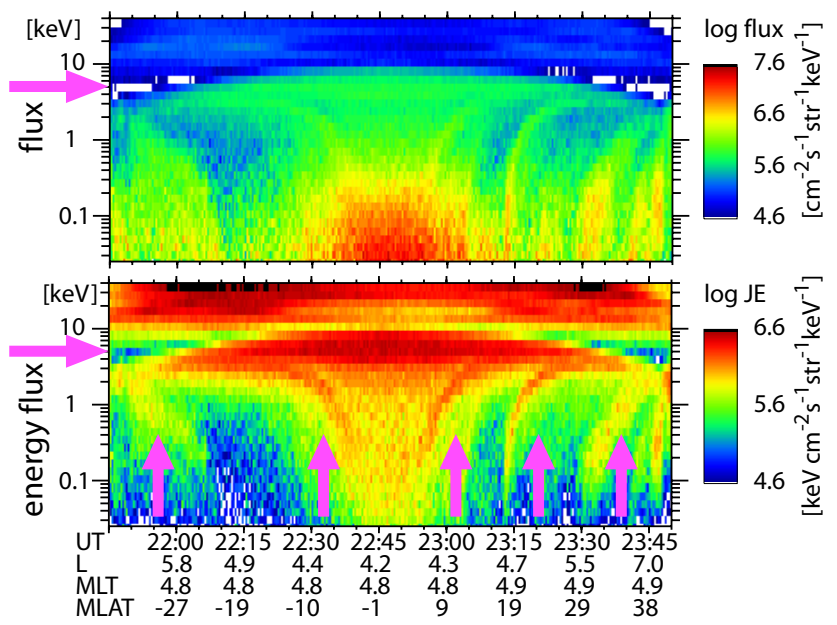

Fig. 2. Same as Fig. 1 except date (22 December 2004), energy range $(0.03-40 \mathrm{keV})$, and color scale. The contamination from $\mathrm{MeV}$ particles of radiation belt is seen at around 22:30 23:00 UT. The band-like structure is marked by horizontal arrows and the wedgelike energy-latitude sub-keV ion structure is marked by vertical arrows. One can recognize clear gap between the band-like structures at high energy $(>10 \mathrm{keV})$ and at low energy $(<5 \mathrm{keV})$.

and e). This motion is known for long time for the bandlike structure (e.g., Ejiri et al., 1980), but not for the wedgelike structure. The superficial controversy between the substorm preference of the wedge-like structure (Yamauchi et al., 2006) and the quiet-time preference of Vallat's nose (Vallat et al., 2007) is explained by this outward shift of the drift shell location during the quiet time. Thus, the statistics confirms that the coexistence of the band-like structure and the wedge-like structure is the feature related to the past substorms. Therefore, one can safely select one of the example classified as Fig. 1b for the simulation work.

\section{Numerical tracing}

In this section we show the numerical result to reconstruct the observation from an assumed ion distribution in the plasma sheet. To assume a proper ion distribution, we backward traced the ions from the actual observation. Thus the method consists of both backward and forward tracing. Backward part is phase space mapping and forward part is ion drift simulation.

\subsection{Selected event}

As mentioned in the previous section, we just need to find a typical example for this study. Here we selected the traversal that shows both the band-like and wedge-like structures independently such that we can clearly separate them from each other. Such example enables us to obtain the relative source density and source temperature of these two structures, and hence to understand the relative contribution to the global circulation of ions.

Figure 2 shows the data we select for the present study. The provisional AE is about 100 200 nT during past several hours with $400 \mathrm{nT}, 800 \mathrm{nT}$, and $1500 \mathrm{nT}$ activities about $6 \mathrm{~h}$, $9 \mathrm{~h}$, and $14 \mathrm{~h}$ before the traversal, respectively. The geomagnetic condition corresponds to the transition between Fig. 1b and $1 \mathrm{c}$, and therefore represents the majority of the cases.

In Fig. 2, one can recognize the wedge-like structure between $0.1 \sim 3 \mathrm{keV}$. While the low energy part of the wedge-like structure $(<0.2 \mathrm{keV})$ lies rather vertical in the spectrogram (change of characteristic energy within short time/distance), the highest energy part $(>2 \mathrm{keV})$ lies horizontal in the spectrogram (nearly the same energy for long time/distance), merging with the band-like structure at several keV. Above this energy, a clear ion cavity is seen at around $10 \mathrm{keV}$. This cavity demarcates the westward drifting energy domain $(>10 \mathrm{keV})$ and the eastward drifting energy domain $(<5 \mathrm{keV})$ for ions. A successful simulation must reproduce all three components: high-energy westward drifting band-like structure, low-energy eastward drifting bandlike structure, and the wedge-like structure.

\subsection{Backward tracing}

We first back-trace the ion distribution given in Fig. 2 using the phase space mapping method assuming that all ions obeys the $\boldsymbol{E} \times \boldsymbol{B}$ and the magnetic drifts under given (timevarying) electric and magnetic fields (e.g., Kistler et al., 1999; Ebihara et al., 2008, and references therein) and given loss rate. For magnetic and electric fields, we use a dipole magnetic field and the Weimer-2001 type convection electric field model (Weimer, 2001) with corotation electric field (solar wind parameters were obtained by using the 5-min resolution NASA/OMNI data set). We use such a simple magnetic field because we do not focus on the exact reconstruction of source distribution function. For the particle loss, we assumed charge exchange decay in the same way as Ebihara et al. (2001). The back-tracing is stopped when either ions reach a given starting boundary $\left(R=8 R_{E}\right.$ in the present case) or elapsed time exceeds a certain time $(34 \mathrm{~h}$ in the present case). The result gives us the source ion distribution in energy-space domain with energy-dependent time lag that corresponds to the elapsed time.

We here assumed the starting boundary at $R=8 R_{E}$ and 90 degree pitch angle. Strictly speaking, we have to set the starting boundary such that the source population does not have energy-time dispersion for all pitch angles. However, this is not a simple task because drift depends on the model electric field for which we have only an empirical model or statistical average. Fortunately, our interest is to reconstruct the temperature and density rather than the fine distribution function of the source ions, and hence, we do not need exact location of the source. Therefore, we employed the boundary 
(a) Back-traced location at $\mathrm{R}=\mathbf{8} \mathrm{R}_{\mathrm{E}}$ from Cluster location

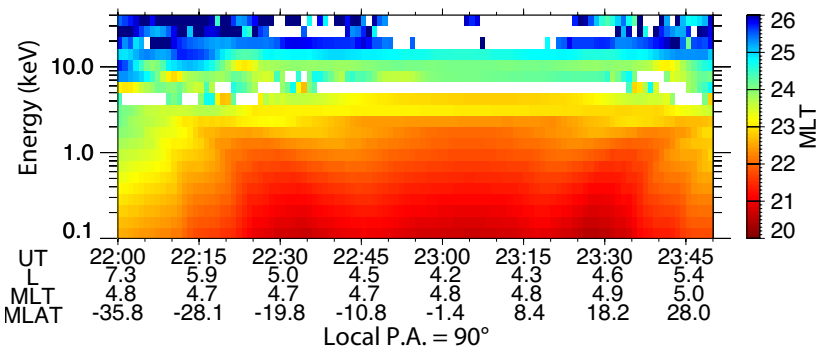

(b) Elapsed from $R=8 R_{E}$ to Cluster location

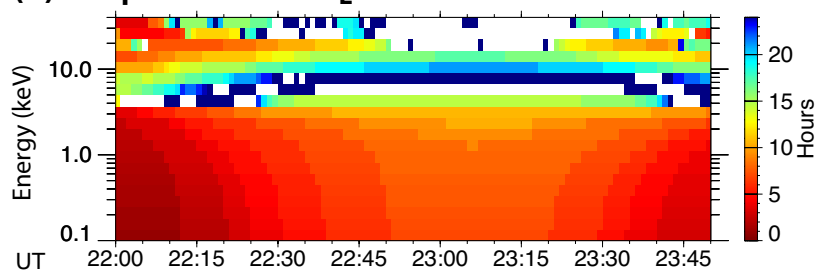

Fig. 3. Back-traced local time (upper panel) and estimated elapsed time (lower panel) from the satellite location to starting boundary at $R=8 R_{E}$ using backward phase-space mapping under assumed electric field and magnetic field (see text for the field model).

location from our past model in which we tried several different boundaries and found one what minimizes the dispersion (Ebihara et al., 2001). If the back-traced source population does not have energy-time dispersion, the assumed boundary is within acceptable error for our purpose of obtaining the density and the temperature of the sources.

Since the Liouville theory guarantees one-to-one mapping in phase space, we can obtain only limited area in location and time for a given energy at the satellite location. To overview such scattering of the back-traced region and time, we examine the back-traced location (local time in this case) and the elapsed time at the starting boundary $\left(R=8 R_{E}\right)$ for all energies of all observation points (i.e., pair of UT and location) independent of the actual observation of ions. Figure $3 \mathrm{a}$ and $\mathrm{b}$ shows the results, respectively.

Ions with different energies are back-traced to different locations and starting time. According to Fig. 3a, the low-energy ions corresponding to the wedge-like part $(<2 \mathrm{keV})$ comes from 20 22 MLT whereas the high-energy ions $(>10 \mathrm{keV})$ comes from 00 02 MLT for all observation points by the satellite if they started from $8 R_{E}$ distance. As for the wedge-like part, the difference in the starting MLTs between $0.1 \mathrm{keV}$ ions and $1 \mathrm{keV}$ ions are about one hour.

According to Fig. $3 \mathrm{~b}$, the low-energy ions $(<2 \mathrm{keV})$ reach the spacecraft location much faster than the high-energy ions $\left(>10 \mathrm{keV}\right.$ ) by several hours if both started from $8 R_{E}$ distance. Even within the same energy domain, the elapsed time at different observation time (eventually latitude) is quite different. For example, the elapsed time for $<2 \mathrm{keV}$ ions observed at 23:10 UT is about $6 \sim 8 \mathrm{~h}$, whereas those observed at 23:30 UT is about $4 \sim 5 \mathrm{~h}$. To make best use of these charac-

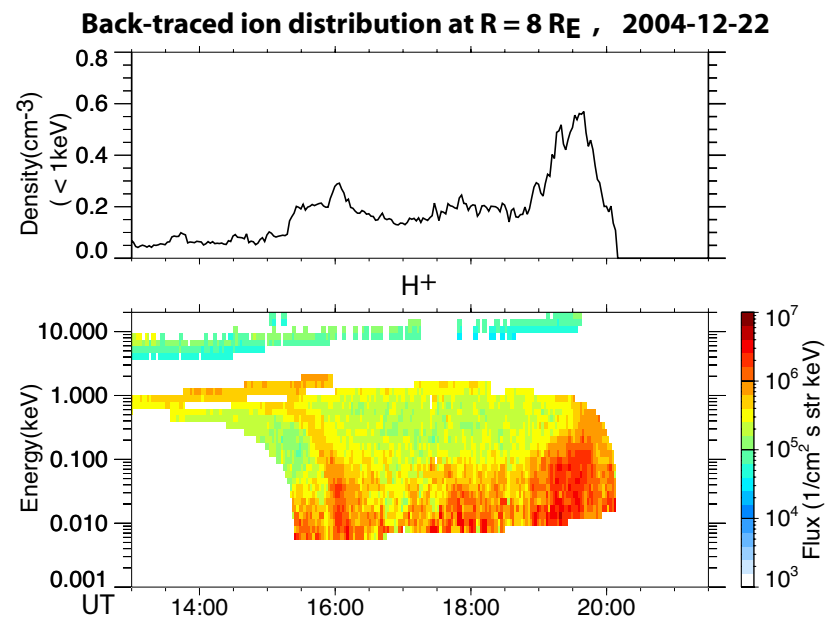

Fig. 4. Number density (upper panel) and energy-time flux spectrogram (lower panel) of the estimated $\mathrm{H}^{+}$source that is obtained by applying the backward phase-space mapping to the observed protons during 23:07 23:40 UT (Southern Hemisphere only) shown in Fig. 2. We did not use the data before 23:07 UT to remove contamination from radiation belt particles. The assumed electric field and magnetic field are described in Sect. 2.

teristics on the elapsed time and mapped MLT, we back-trace only a limited time period from data shown in Fig. 2.

Figure 4 shows the result of backward phase-space mapping of the observed protons by CIS during 23:07 23:40 UT (Northern Hemisphere) shown in Fig. 2 using the model mentioned above. One hemisphere data is enough to reconstruct the source distribution that is responsible for both hemisphere in the dipole field. The data before 23:07 UT is not used to remove the contamination from the radiation belt $\mathrm{MeV}$ particles in the middle of the traversal, although such limitation in the data automatically excludes data corresponding to the second and third stripes of the wedge-like structure in Fig. 2 (22:25 22:40 UT and 22:55 23:05 UT, respectively).

Since the elapsed time for $<2 \mathrm{keV}$ ions and that for $>3 \mathrm{keV}$ ions are quite different (Fig. 3b), the time span shown in Fig. 4 (after 15:20UT) corresponds only to the $<2 \mathrm{keV}$ ions. In this way, we eventually selected only the wedge-like part to obtain the source distribution after 15:20 UT. Note that this method cannot completely filter out $>3 \mathrm{keV}$ ions (e.g. $20 \mathrm{keV}$ at 23:40 UT in Fig. 3b), and hence isolated signatures at high-energy domain $>4 \mathrm{keV}$ $(13: 00 \sim 19: 30 \mathrm{UT})$ and at $1 \sim 2 \mathrm{keV}(13: 00 \sim 16: 00 \mathrm{UT})$ remained in Fig. 4. We treat this as artifacts of the backward phase-space mapping.

Figure 4 shows several features that are common with those reported in Ebihara et al. (2008), in which the same tracing method was applied to the Equator-S data. (1) The hot $1 \sim 3 \mathrm{keV}$ ion at the Cluster location are mostly mapped to low energy $<0.2 \mathrm{keV}$ at the starting boundary. These 


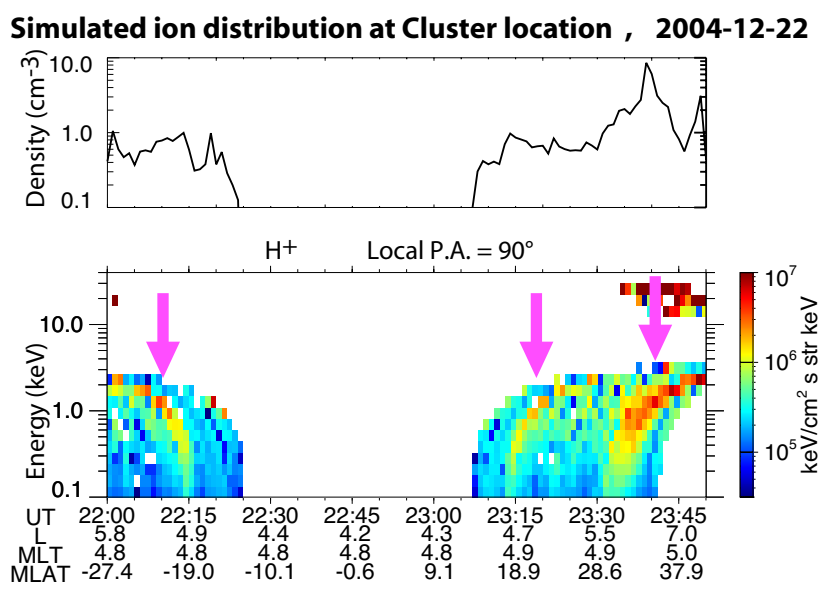

Fig. 5. Number density (upper panel) and energy-time energy flux spectrogram (lower panel) of simulated $\mathrm{H}^{+}$using drift model starting from an estimated source distribution, which is obtained, by the power-law fitting of the distribution in Fig. 4, and by assuming uniform over the entire MLT in the source region (all nightside). The wedge-like structure is marked by vertical arrows. The model is described in Sect. 3 and Ebihara et al. (2004, 2008).

cold ions adiabatically gain the energy by the electric field, and form the wedge-like dispersed part. (2) Fine dispersive stripes of the wedge-like structure disappeared at the starting boundary except for one stripe before 16:00 UT. Instead, we have smooth enhancement of cold ions: one at around 19:00 20:00 UT corresponds to the fifth stripe (23:30 23:40 UT) in Fig. 2, and the other at around 15:30 16:30 UT corresponds to the fourth stripe (23:12 23:25 UT) in Fig. 2. The dispersion-free distribution without fine structures supports the rational of our boundary as within acceptable error for our purpose of obtaining the source density and temperature. (3) The estimated source ions are cold with temperature $\leq 0.1 \mathrm{keV}$. This is far below the typical plasma sheet temperature. (4) Density of this source cold ions corresponding to the wedge-like structure is about $0.2 \sim 0.5 \times 10^{6} / \mathrm{m}^{3}$.

\subsection{Forward simulation}

The back-traced source consists of the minimum amount of ions that are needed to cause the observed ion distribution during the traversal because the one-to-one mapping in the phase space means that backward mapping method misses the source component that never reaches or overshoots the spacecraft location at the time of the observation. To fill this gap, we need some sort of interpolation. To confirm the interpolated source distribution to be adequate, we double-check the estimated source and location by further simulating forward.

The source distribution for the simulation is obtained in the same manner as Ebihara et al. (2008). We first power- law fit the source distribution given in Fig. 4, and then assume uniform distribution over all MLTs and all pitch angles in the source region $\left(R=8 R_{E}\right.$ nightside). Using this smoothed source distribution, we simulated ion drift forward up to the Cluster location and time. The detailed scheme of the forward simulation including the assumed decay rate is described in Ebihara et al. (2004).

The results shown in Fig. 5 are summarized as follows. (1) The first, fourth, and fifth stripes of the wedge-like structure in Fig. 2 are reconstructed from the assumed cold ions given in Fig. 4, although we used only the fourth and fifth stripes for the model. The source cold ions are adiabatically energized in the nightside, and reached the spacecraft location by the eastward drift. (2) No ion is seen at the energy of bandlike structure which has quite different elapsed time $(>10 \mathrm{~h})$ from the wedge-like part. (3) Contamination at the middle of traversal in Fig. 2 is removed. This is the trade off of ignoring the third and fourth stripes in Fig. 2. (4) The density at the Cluster location increased to about $1 \sim 5 \times 10^{6} / \mathrm{m}^{3}$, by a factor of about $5 \sim 10$ from the source (Fig. 4). Note that the isolated signature at around $20 \mathrm{keV}$ after 23:35 UT in Fig. 5 is the result of what we treat as the artifact in Fig. 4, and hence we ignore them.

Corresponding to the band-like structure at several $\mathrm{keV}$, we assumed an isotropic Maxwellian plasma with constant temperature $(1 \mathrm{keV})$ and constant density $\left(0.5 \times 10^{6} / \mathrm{m}^{3}\right)$ as the source plasma (at $R=8 R_{E}$ ). The assumed density is slightly higher than that of the cold plasma shown in Fig. 4. This plasma sheet component drifts both westward and eastward depending on how much they are adiabatically energized in the nightside (energization depends on MLT) before reaching the drift shell. The ions that stays $<5 \mathrm{keV}$ drift eastward, whereas ions that are energized to $>10 \mathrm{keV}$ drift westward. If the plasma sheet hot source exists very long time, all the three components (both the band-like structures and the wedge-like structure) may meet simultaneously at the Cluster location. Such case is shown in Fig. 6.

Figure 6 shows the result of the drift simulation including both contributions from the hot component and the cold component shown in Fig. 5. Two band-like structures are added to Fig. 5: the high-energy part ( $>10 \mathrm{keV})$ comes mainly from the pre-midnight sector via westward drift, whereas the lowenergy part $(<5 \mathrm{keV})$ comes mainly from the post-midnight sector via eastward drift. Thus, Fig. 6 reproduced the general morphology of the CIS observation (Fig. 2) for all three components: both the band-like structures including a gap and the wedge-like structure that overlaps the low-energy band-like structure. The result confirms that we just need cold $(<0.1 \mathrm{keV})$ plasma and hot $(\sim 1 \mathrm{keV})$ plasma in the nearEarth magnetotail to explain the CIS observation at the entire energy range $(0.1 \sim 40 \mathrm{keV})$.

The enhancement of the hot component from the starting boundary $\left(0.5 \times 10^{6} / \mathrm{m}^{3}\right)$ to the observation point (about $1 \times 10^{6} / \mathrm{m}^{3}$ ) is only by a factor 2 , which is less than the enhancement of the cold component (by a factor of $>5$ ) that 
corresponds to the wedge-like structure. The less enhancement rate of the low-energy band-like part is partly because (1) the charge-exchange loss is much larger for $\mathrm{keV}$ ions than sub-keV ions with longer elapsed time and large chargeexchange rate for $\mathrm{keV}$ ions than sub-keV ions, and because (2) only a part of the hot plasma drifts eastward whereas majority of the cold plasma drifts eastward. Without the chargeexchange loss, density enhancement of the eastward drifting $\mathrm{keV}$ ions might be larger than that of sub-keV ions because the eastward ion motion is decelerated by the westward magnetic drift which is proportional to the ion energy.

\section{Discussion}

The cold source for the wedge-like structure is already demonstrated in Ebihara et al. (2008), and hot source for the eastward drifting band-like structure is already demonstrated in Vallat et al. (2007). However, no simulation has produced three components (the wedge-like structure, the overlapping low-energy band-like structure, and the high-energy bandlike structure) in a single condition, i.e., in the same timedependent electric field that is given independently from the solar wind condition. In this sense, this is the first confirmation of multiple source temperatures (hot $\sim 1 \mathrm{keV}$ and cold $<0.1 \mathrm{keV}$ ) in a model independent way. The wedge-like structure and the band-like structure are two different phenomena although they overlap to each other. In other words, they could be separated in the noon sector where the highest energy of the wedge-like structure is below the energy of the band-like structure, although no statistical works has been performed in searching eastward drifting band-like structure that is separated from the wedge-like structure.

As mentioned in Sect. 2, we assumed uniformness of ion distribution function in the MLT direction at a given UT shown in Fig. 3. Since we cannot derive the ion sources that does not reach or overshoot the satellite location at the observation UT, the source could be different from the one shown in Fig. 3. However, such ambiguity should not affect the main indication that the wedge-like dispersed part comes from cold ions. Since the wedge-like structure is the fossil of substorms (Table 1 and Yamauchi and Lundin, 2006), supply mechanism of such cold ions needs to be identified. Recently, Engwall et al. (2008) found large field-aligned escape of cold ions from the ionosphere but its relation to the geomagnetic activity is not clear.

The band-like $\mathrm{keV}$ ions are also visible in Viking data (e.g., Figs. 2 and 6 in Ebihara et al., 2001), but we could not report it because this band-like structure and the wedge-like structure were detected by different instruments (PISP-1 for $1 \sim 40 \mathrm{keV}$ ions and PISP-2 for $0.05 \sim 0.5 \mathrm{keV}$ ions, respectively). Since sensitivities of PISP-1 and PISP-2 are quite different, we could not judge if this band-like structure is physically independent of the wedge-like structure or a part of the wedge-like structure.
Simulated ion distribution at Cluster location , 2004-12-22
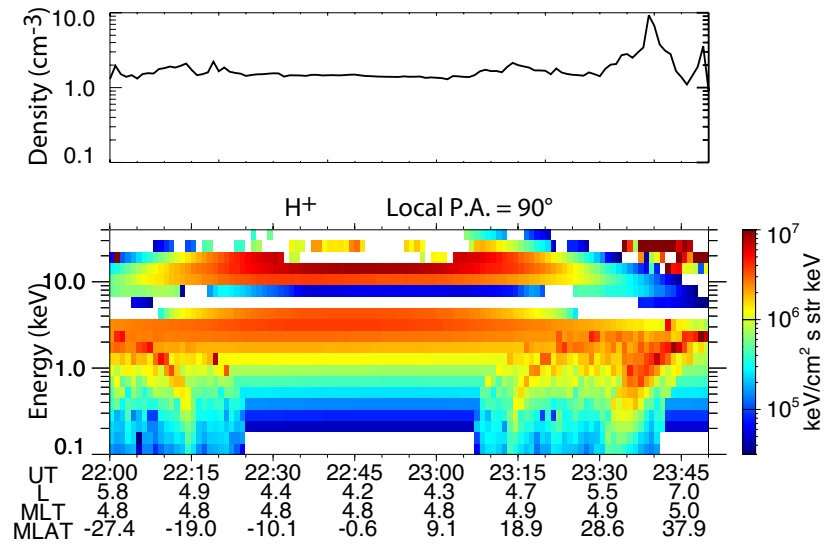

Fig. 6. Number density (upper panel) and energy-time energy flux spectrogram (lower panel) of simulated $\mathrm{H}^{+}$starting from the summation of cold source (Fig. 5) and hot source (Maxwellian with $1 \mathrm{keV}$ temperature and $0.5 \times 10^{6} / \mathrm{m}^{3}$ density). The hot source is assumed to be constant all the time. The assumed electric field and magnetic field are described in Sect. 2.

The present result gives new interpretation of our old statistics in the MLT distribution of the wedge-like structure (Fig. 3 of Yamauchi et al., 2006): the sub-group of the wedge-like structure that "extends to $>1 \mathrm{keV}$ " contains both plasma sheet ions and cold ions. Note that the old conclusions from this statistics are not affected because we used it as the MLT distribution of the wedge-like structure. The obvious future task is to find the MLT distribution of the eastward drifting band-like ions and the history of this MLT distribution after substorms.

\section{Conclusion}

Out of more than 100 Cluster morning (02 07 LT) traversals, about $90 \%$ of the traversals show the combination of the wedge-like structure and the overlapping band-like structure in the CIS ion spectrogram. The correlation study with the provisional $\mathrm{AE}$ confirmed that this overlapping combination is associated with the past substorm activities, in the similar way as Yamauchi and Lundin (2006). The numerical tracing methods (backward phase-space mapping and forward simulation) indicate that these two components and the highenergy ions originate from two source populations: cold and hot. The wedge-like dispersed ions come from cold ions of $<0.1 \mathrm{keV}$ in the nightside, and the band-like ions (both the high-energy part above the cavity and the low-energy parts below the cavity) come from plasma sheet hot ions $\sim 1 \mathrm{keV}$. The estimated source densities are about $0.2 \sim 0.5 \times 10^{6} / \mathrm{m}^{3}$ for the cold part and about $0.5 \times 10^{6} / \mathrm{m}^{3}$ for the hot part, although the Cluster observation shows slightly higher density for the wedge-like part than for the low-energy band-like 
part. The wedge-like part has much shorter elapsed time, i.e., less charge-exchange loss, than the band-like part.

Acknowledgements. The provisional $\mathrm{AE}$ index is provided by WDC-C2 for geomagnetism at Kyoto University. The solar wind OMNI data were obtained from the GSFC/SPDF OMNIWeb interface. The Cluster project is managed by European Space Agency (ESA). The work is partly supported by Swedish National Space Board (RS) and by Swedish Research Counsel (VR) \#621-20055005. Ebihara's part of the work is partly supported by the Program for Improvement of Research Environment for Young Researchers through the Special Coordination Funds for Promoting Science and Technology (SCF) commissioned by the Ministry of Education, Culture, Sports, Science and Technology (MEXT) of Japan. Yamauchi thanks programs for disabled people in Sweden which have made it possible for him to work.

Topical Editor R. Nakamura thanks P. Newell and another anonymous referee for their help in evaluating this paper.

\section{References}

Alfvén, H. and Fälthammar, C.-G.: Cosmical Electrodynamics, Fundamental Principles, Clarendon, Oxford, 1963.

Ebihara, Y., Yamauchi, M., Nilsson, H., Lundin, R., and Ejiri, M.: Wedge-like dispersion of sub-keV ions in the dayside magnetosphere: Particle simulation and Viking observation, J. Geophys. Res., 106, 29571-29584, 2001.

Ebihara, Y., Ejiri, M., Nilsson, H., Sandahl, I., Grande, M., Fennell, J. F., Roeder, J. L., Weimer, D. R., and Fritz, T. A.: Multiple discrete-energy ion features in the inner magnetosphere: 9 February 1998, event, Ann. Geophys., 22, 1297-1304, 2004, http://www.ann-geophys.net/22/1297/2004/.

Ebihara, Y., Kistler, L. M., and Eliasson, L.: Imaging cold ions in the plasma sheet from the Equator-S satellite, Geophys. Res. Lett., 35, L15103, doi:10.1029/2008GL034357, 2008.

Ejiri, M., Hoffman, R. A., and Smith, P. H.: Energetic particle penetration into the inner magnetosphere, J. Geophys. Res., 85(A2), 653-663, 1980.

Engwall, E., Eriksson, A. I., Cully, C. M., André, M., Torbert, R., and Vaith, H.: Earth's ionospheric outflow dominated by hidden cold plasma, Nature Geoscience, 2, 24-27, doi:10.1038/ngeo387, 2009.

Kistler, L. M., Klecker, B., Jordanova, V. K., Mbius, E., Popecki, M. A., Patel, D., Sauvaud, J. A., Rme, H., Di Lellis, A. M., Korth, A., McCarthy, M., Cerulli, R., Bavassano Cattaneo, M. B., Eliasson, L., Carlson, C. W., Parks, G. K., Paschmann, G., Baumjohann, W., and Haerendel, G.: Testing electric field models using ring current ion energy spectra from the Equator-S ion composition (ESIC) instrument, Ann. Geophys., 17, 1611-1621, 1999, http://www.ann-geophys.net/17/1611/1999/.
Kovrazhkin, R. A., Sauvaud, J.-A., and Delcourt, D. C.: INTERBALL-Auroral observations of $0.1-12 \mathrm{keV}$ ion gaps in the diffuse auroral zone, Ann. Geophys., 17, 734-742, 1999, http://www.ann-geophys.net/17/734/1999/.

Lennartsson, W., Shelley, E. G., Sharp, R. D., Johnson, R. G., and Balsiger, H.: Some initial ISEE-1 results on the ring current composition and dynamics during the magnetic storm of December 11, 1977, Geophys. Res. Lett., 6(6), 483-486, 1979.

Newell, P. T. and Meng, C. I.: Substorm introduction of $\mathfrak{x} 1 \mathrm{keV}$ magnetospheric ions into the inner plasmasphere, J. Geophys. Res., 91, 11133-11145, 1986.

Quinn, J. M. and McIlwain, C. E.: Bouncing ion clusters in the Earth's magnetosphere, J. Geophys. Res., 84, 7365-7370, 1979.

Rème, H., Aoustin, C., Bosqued, J. M., et al.: First multispacecraft ion measurements in and near the Earth's magnetosphere with the identical Cluster ion spectrometry (CIS) experiment, Ann. Geophys., 19, 1303-1354, 2001, http://www.ann-geophys.net/19/1303/2001/.

Sauvaud, J. A., Crasnier, J., Mouala, K., Kovrazhkin, R. A., and Jorjio, N. V.: Morning sector ion precipitation following substorm injections, J. Geophys. Res., 86, 3430-3438, 1981.

Seki, K., Hirahara, M., Hoshino, M., Terasawa, T., et al.: Cold ions in the hot plsma sheet of Earth's magnetotail, Nature, 422(6932), 589-592, 2003.

Vallat, C., Ganushkina, N., Dandouras, I., Escoubet, C. P., Taylor, M. G. G. T., Laakso, H., Masson, A., Sauvaud, J.-A., Rème, H., and Daly, P.: Ion multi-nose structures observed by Cluster in the inner Magnetosphere, Ann. Geophys., 25, 171-190, 2007, http://www.ann-geophys.net/25/171/2007/.

Weimer, D.: An improved model of ionospheric electric potentials including substorm perturbations and application to the Geospace Environment Modeling November 24, 1996, event, J. Geophys. Res., 106, 407-416, 2001.

Yamauchi, M., Lundin, R., Eliasson, L., and Norberg, O.: Mesoscale structures of radiation belt/ring current detected by lowenergy ions, Adv. Space Res., 17(2), 171-174, 1996.

Yamauchi, M., Lundin, R., Eliasson, L., Winningham, D., Reme, H., et al.: Structures of sub-keV ions inside the ring current region, in: The Inner Magnetosphere: Physics and Modeling, edited by: Pulkkinen, T. I., Tsyganenko, N. A., and Friedel, R., AGU Monograph, 41-46, 2005.

Yamauchi, M. and Lundin, R.: Sub-keV ring current ions as the tracer of substorm injection, Ann. Geophys., 24, 355-366, 2006, http://www.ann-geophys.net/24/355/2006/.

Yamauchi, M., Brandt, P. C., Ebihara, Y., Dandouras, I., Nilsson, H., et al: Source location of the wedge-like dispersed ring current in the morning sector during a substorm, J. Geophys. Res., 111, A11S09, doi:10.1029/2006JA011621, 2006. 